\title{
Movilidad urbana en el Campus Central de la Universidad de Panamá: Caso de la Facultad de Arquitectura y Diseño y la Facultad de Ciencias de la Educación
}

\author{
Urban mobility in the Main Campus of the University of Panama: \\ Case of the Faculty of Architecture and the Faculty of Education \\ Sciences
}

\author{
Ángela Castañeda ${ }^{l}$, Gigiola Ocampo ${ }^{l}$, Karen Sánchez ${ }^{l}$, Jorge Peren ${ }^{2 *}$ \\ ${ }^{1}$ Facultad de Arquitectura y Diseño, Universidad de Panamá, Ciudad de Panamá, Panamá., \\ ${ }^{2}$ Sustainable Building and City Research Group - SusBCity, Ciudad de Panamá, Panamá
}

\begin{abstract}
Resumen La población del Campus Central de la Universidad de Panamá (UP) es de 25,824 estudiantes, 1,500 profesores y 1,445 administrativos aproximadamente. La movilidad urbana de los usuarios puede tener un impacto en su rutina diaria y en su calidad de vida. El objetivo del presente trabajo es estudiar la movilidad urbana de los usuarios de dos facultades del campus central de la UP: (1) la Facultad de Arquitectura y Diseño y (2) la Facultad de Ciencias de la Educación y realizar una comparación entre ambas facultades que sirva de base para futuras políticas de movilidad en el campus universitario de la UP. Se aplicó una encuesta al 15\% de los estudiantes, administrativos y profesores de ambas facultades. Los resultados destacan que para la Facultad de Arquitectura y Diseño la mayoría de su población reside en Bethania (11.48\%), Bella Vista (9.84\%) y Juan Díaz (9.43\%); los medios de transporte más usados son: (metro y taxi, bus y carro, etc.), automóvil y bus. Para la Facultad de Ciencias de la Educación, los tres corregimientos con más población del campus son Bethania (10.13\%), Juan Díaz (10.13\%) y Tocumen $(8.23 \%)$; los medios de transporte más usados son: (metro y taxi, bus y carro, etc.), metro bus y bus.
\end{abstract}

Palabras claves Ciudades, campus universitario, movilidad urbana, transporte.

\begin{abstract}
The population of the Central Campus of the University of Panama (UP) is 25,824 students, 1,500 professors and 1,445 businesses approximately. The urban mobility of users can have an impact on their daily routine and their quality of life. The objective of the work is the urban mobility of the users of the Faculties of the UP central campus: (1) the Faculty of Educational Sciences and make a comparison between both Faculties that serves as a basis for future mobility policies on campus University of the UP. A survey was applied to $15 \%$ of the students, administrators and professors of both Faculties. The most outstanding results for the Faculty of Architecture and Design of its population reside in Bethania (11.48\%), Bella Vista (9.84\%) and Juan Díaz (9.43\%); The most used means of transport are: (metro and taxi, bus and car, etc.), car and bus. For the Faculty of Education Sciences, the three villages with the most population of the campus are Bethania (10.13\%), Juan Díaz (10.13\%) and Tocumen $(8.23 \%)$; The most used means of transport are: (metro and taxi, bus and car, etc.), metro bus and bus.
\end{abstract}

Keywords Cities, university campus, transportation, urban mobility.

\footnotetext{
*Corresponding author: isaacperen@ hotmail.com
}

\section{Introducción}

El desarrollo de las áreas urbanas hace surgir nuevos factores como la falta de un transporte que abastezca la demanda, estructuras viales mal diseñadas en comparación a la cantidad de personas.
La movilidad urbana se puede entender como movimiento o desplazamiento de personas y mercancías o el simple desplazamiento de entes que se efectúa en la ciudad; Movilidad según el diccionario de Filosofía [1] este cambio o proceso de cualquier especie, lo cual explica que es una necesidad el hecho de movilizarnos de lugar en lugar. Esta en 


\section{Castañeda (et al): Movilidad urbana en el Campus Central de la Universidad de Panamá: Caso de la Facultad de Arquitectura y Diseño y la Facultad de Ciencias de la Educación}

sí no es un problema, pero pasa a serlo cuando no se puede realizar de la forma adecuada, óptima o eficaz.

Según el Banco Mundial de Desarrollo [2], Panamá es uno de los países que ha adoptado el automóvil como medio principal de transporte. Este medio es totalmente ineficaz en las ciudades y debido a esto se han creado los difíciles problemas de movilidad. En el país hay pocas calles y de tamaños reducidos por lo cual transportarse de forma individual como se realiza en mayor parte actualmente resulta ineficiente. De acuerdo al Observatorio de Movilidad Urbana [3], en otros sitios como Rio de Janeiro y Manaos se ha optado por el uso mayoritario de transporte colectivo y a pie.

El propósito de esta investigación es evaluar la movilidad urbana en el campus universitario, a través del estudio sobre el tipo de transporte que usan los encuestados así como el tiempo, sitios de las residencias entre otros. Estos datos posibilitarán futuros análisis sobre el desplazamiento de los usuarios de dicha institución. Hasta el momento no existe ningún documento de referencia en el cual se haya realizado este tipo de trabajo, por lo tanto los datos recabados son valiosos para estudios futuros.

La figura 1 A muestra el mapa del campus de la UP con la localización de ambas facultades. La figura $1 \mathrm{~B}$ y la 1C muestran fotos de ambas facultades.

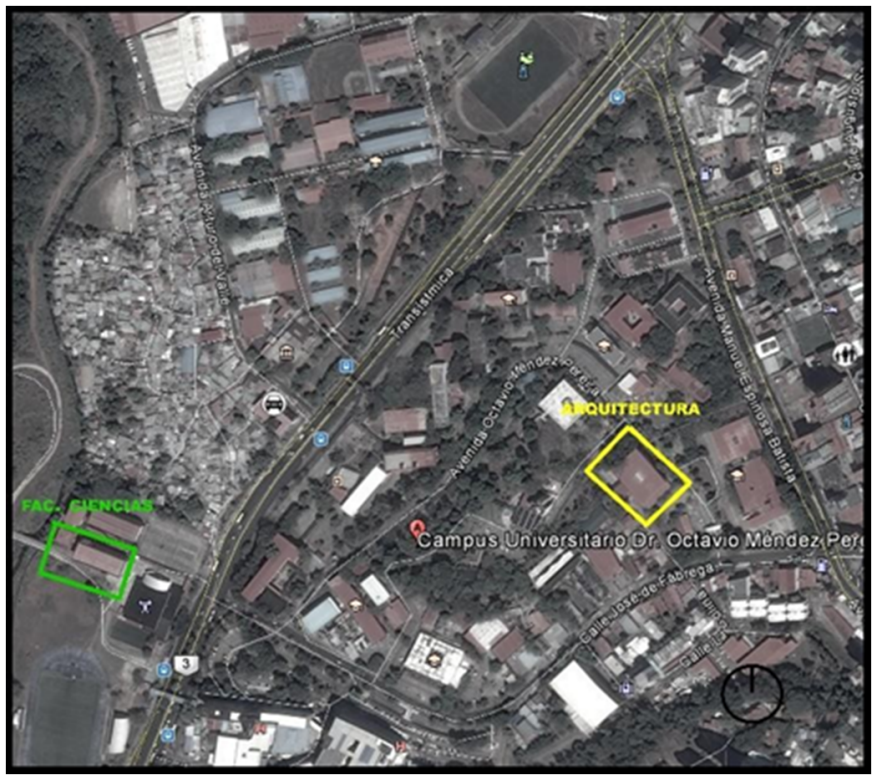

Figura 1 A. Localización. Fuente: Google Earth Pro.

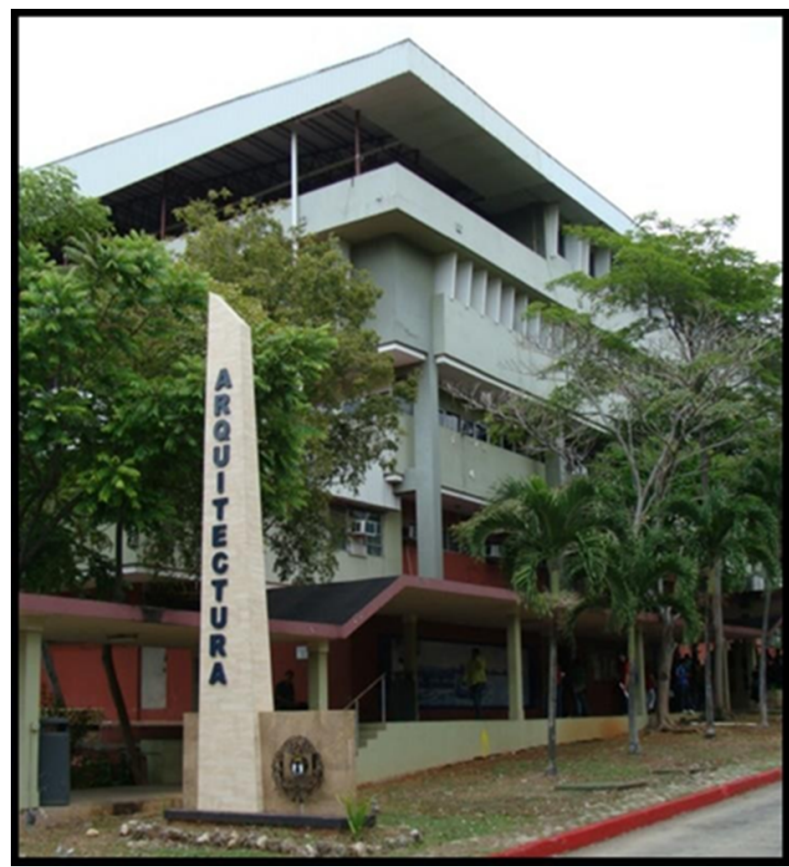

Figura 1 B. Facultad de Arquitectura y Diseño. Fuente: http://www.up.ac.pa

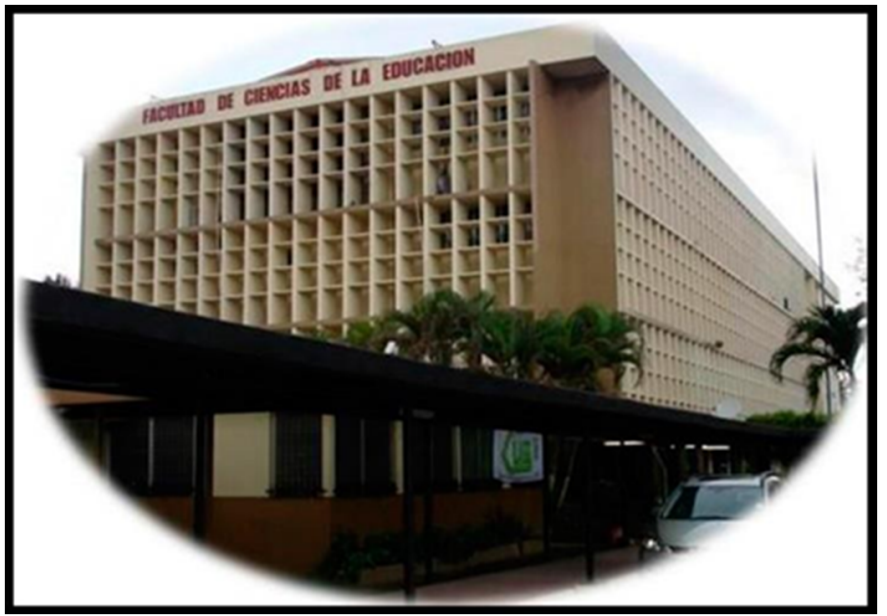

Figura 1 C. Facultad de Ciencias de la Educación. Fuente: http://www.up.ac.pa

\section{Objetivos}

- Realizar un estudio del tipo de transporte que los estudiantes de la Universidad de Panamá, especialmente de la Facultad de Arquitectura y Diseño y la Facultad de Ciencias de la Educación, utilizan para movilizarse hasta el campus central.

- Lograr que una vez los datos sean terminados y ordenados, hacerlos públicos para que estos sirvan como ejes para trabajos futuros; así como comparaciones de próximas encuestas sobre Movilidad Urbana que se lleguen a realizar. 
Castañeda (et al): Movilidad urbana en el Campus Central de la Universidad de Panamá: Caso de la Facultad de Arquitectura y Diseño y la Facultad de Ciencias de la Educación

\section{Metodología}

Para realizar este estudio se empleó la siguiente metodología: (1) Se identificó la cantidad de estudiantes, profesores y administrativos por cada facultad para saber cuán grande debía ser esta investigación; (2) Se evaluaron las opciones de herramientas o plataformas de encuestas: Survey Monkey, Online Encuestas, Question Pro, Survio, Google Forms y Eval \& Go, de las cuales surgió como mejor plataforma Online Encuestas; (3) Se revisó la encuesta y los datos iniciales formulados por el equipo de SusBCity del 2016; (4) Se revisaron y formularon nuevas preguntas con el objetivo de mejorar la encuesta; (5) Se obtuvieron los permisos de diferentes profesores para realizar la encuesta en 10 minutos de sus clases; (6) Se aplicó la encuesta en ambas facultades empleando dispositivos móviles, redes sociales y panfletos impresos. En arquitectura se realizaron las encuestas según los permisos de los profesores mientras que en la Facultad de Ciencias de la Educación se planificó un día específico gracias a la obtención de un permiso con la dirección de dicha facultad.

\section{Resultados}

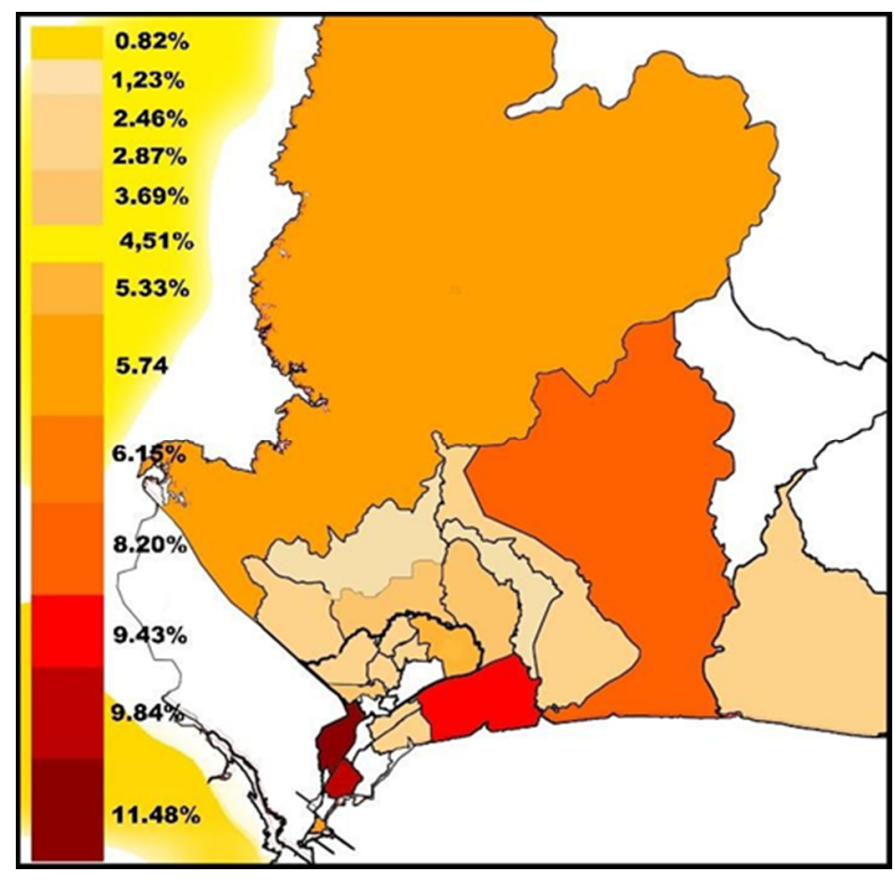

Figura 2 A. Facultad de Arquitectura y Diseño.
Tabla 1. Facultad de Arquitectura y Diseño

\begin{tabular}{|l|l|l|l|}
\hline SAN FELIPE & $0 \%$ & $\begin{array}{l}\text { AMELIA DENIS DE } \\
\text { ICAZA }\end{array}$ & $2.87 \%$ \\
\hline EL CHORRILLO & 0.825 & BELISARIO PORRAS & $2.05 \%$ \\
\hline SANTA ANA & $0.82 \%$ & $\begin{array}{l}\text { JOSÉ DOMINGO } \\
\text { ESPINAR }\end{array}$ & 5.74 \\
\hline $\begin{array}{l}\text { LA EXPOSICIÓN O } \\
\text { CALIDONIA }\end{array}$ & $0 \%$ & MATEO ITURRALDE & $0 \%$ \\
\hline CURUNDÚ & $0 \%$ & VICTORIANO LORENZO & $0 \%$ \\
\hline BETANIA & $11.48 \%$ & ARNULFO ARIAS & $2.46 \%$ \\
\hline BELLA VISTA & $9.84 \%$ & BELISARIO FRÍAS & $2.46 \%$ \\
\hline PUEBLO NUEVO & $2.46 \%$ & OMAR TORRIJOS & $2.87 \%$ \\
\hline SAN FRANCISCO & $4.51 \%$ & RUFINA ALFARO & $0 \%$ \\
\hline PARQUE LEFEVRE & $2.05 \%$ & PACORA & $0 \%$ \\
\hline RIO ABAJO & $2.05 \%$ & SAN MARTIN & $0 \%$ \\
\hline JUAN DÍAZ & $9.43 \%$ & TOCUMEN & $2.05 \%$ \\
\hline PEDREGAL & $3.69 \%$ & MAÑANITAS & $1.23 \%$ \\
\hline ANCÓN & $1.23 \%$ & 24 DE DICIEMBRE & $8.20 \%$ \\
\hline CHILIBRE & $5.33 \%$ & ERNESTO CÓRDOBA & $3.69 \%$ \\
\hline LAS CUMBRES & $2.46 \%$ & ALCALDE DÍAZ & $1.64 \%$ \\
\hline
\end{tabular}

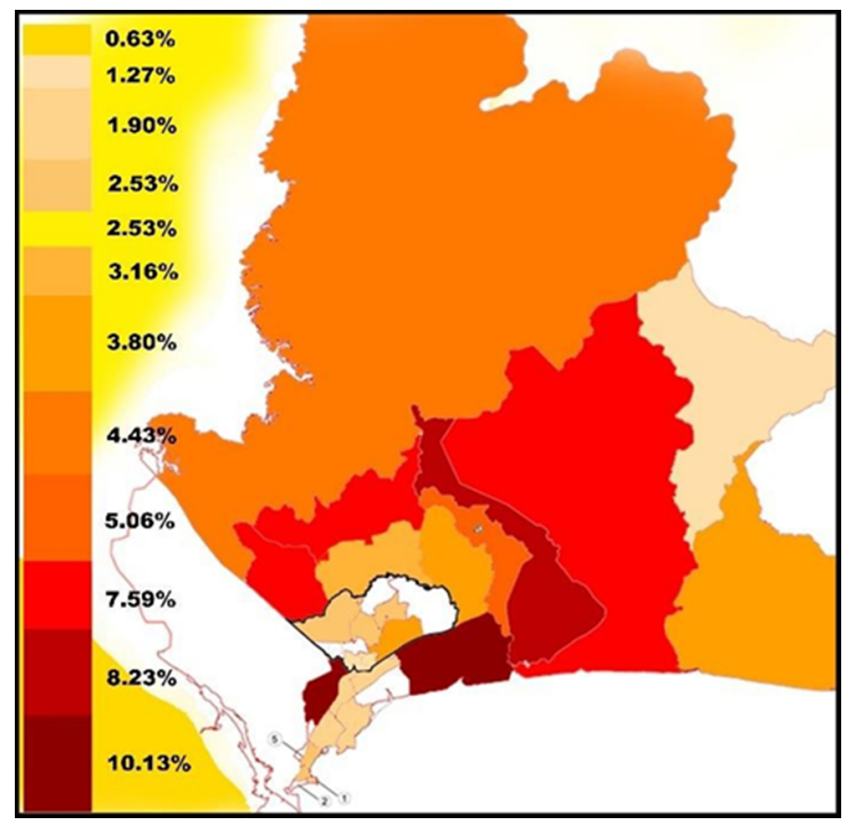

Figura 2 B. Facultad de Ciencias de la Educación. 
Castañeda (et al): Movilidad urbana en el Campus Central de la Universidad de Panamá: Caso de la Facultad de Arquitectura y Diseño y la Facultad de Ciencias de la Educación

Tabla 2. Facultad de Ciencias de la Educación

\begin{tabular}{|l|l|l|l|}
\hline SAN FELIPE & $1.27 \%$ & $\begin{array}{l}\text { AMELIA DENIS DE } \\
\text { ICAZA }\end{array}$ & $0 \%$ \\
\hline EL CHORRILLO & $0 \%$ & BELISARIO PORRAS & $3.16 \%$ \\
\hline SANTA ANA & $1.90 \%$ & $\begin{array}{l}\text { JOSÉ DOMINGO } \\
\text { ESPINAR }\end{array}$ & 4.43 \\
\hline $\begin{array}{l}\text { LA EXPOSICIÓN O } \\
\text { CALIDONIA }\end{array}$ & $1.90 \%$ & MATEO ITURRALDE & $0.63 \%$ \\
\hline CURUNDÚ & $0 \%$ & VICTORIANO & $0.63 \%$ \\
\hline BETANIA & $10.13 \%$ & $\begin{array}{l}\text { ARNULFO ARIAS } \\
\text { MORENZO }\end{array}$ & $1.90 \%$ \\
\hline BELLA VISTA & $1.27 \%$ & BELISARIO FRÍAS & $1.90 \%$ \\
\hline PUEBLO NUEVO & $1.27 \%$ & OMAR TORRIJOS & $1.90 \%$ \\
\hline SAN FRANCISCO & $1.27 \%$ & RUFINA ALFARO & $0 \%$ \\
\hline PARQUE LEFEVRE & $0 \%$ & PACORA & $3.80 \%$ \\
\hline RIO ABAJO & $1.27 \%$ & SAN MARTIN & $0.63 \%$ \\
\hline JUAN DÍAZ & $10.13 \%$ & TOCUMEN & $8.23 \%$ \\
\hline PEDREGAL & $3.16 \%$ & MAÑANITAS & $5.06 \%$ \\
\hline ANCÓN & $3.89 \%$ & 24 DE DICIEMBRE & $7.59 \%$ \\
\hline CHILIBRE & $4.43 \%$ & ERNESTO CÓRDOBA & $2.53 \%$ \\
\hline LAS CUMBRES & ALCALDE DÍAZ & $7.59 \%$ \\
\hline
\end{tabular}

Para la Facultad de Arquitectura y Diseño, los resultados más notorios fueron que un $67 \%$ de los participantes son de la ciudad de Panamá, $26.08 \%$ de Panamá Oeste y un $6.11 \%$ de Colón, de los cuales se dio que los corregimientos con mayor cantidad de encuestados son Bethania con un $11.48 \%$, Bella Vista $9.84 \%$ y Juan Díaz con un $9.43 \%$. (Figura 2 A).

En el caso de la Facultad de Ciencias de la Educación, un 65.7\% de los encuestados son de la Ciudad de Panamá, un 26.91\% son de Panamá Oeste y un 7.4\% son de Colón; para los encuestados por corregimientos los resultados son Bethania con un $10.13 \%$, Juan Díaz con un $10.13 \%$ y Tocumen con $8.23 \%$. (Figura $2 \mathrm{~B}$ ).

La cantidad de estudiantes en la Facultad de Arquitectura y Diseño es de 3,000, 132 profesores y 44 administrativos. La cantidad de encuestados para la Facultad de Arquitectura y Diseño fueron de 358 participantes; de los cuales 331 son estudiantes (12\% de cantidad de estudiantes), 20 profesores (15\%) y 7 administrativos (15\%).

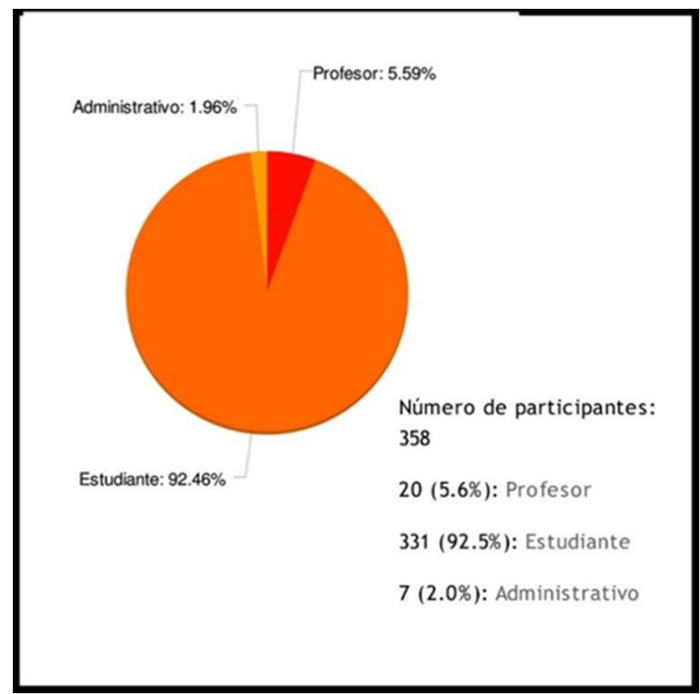

Figura 3 A. Facultad de Arquitectura y Diseño.

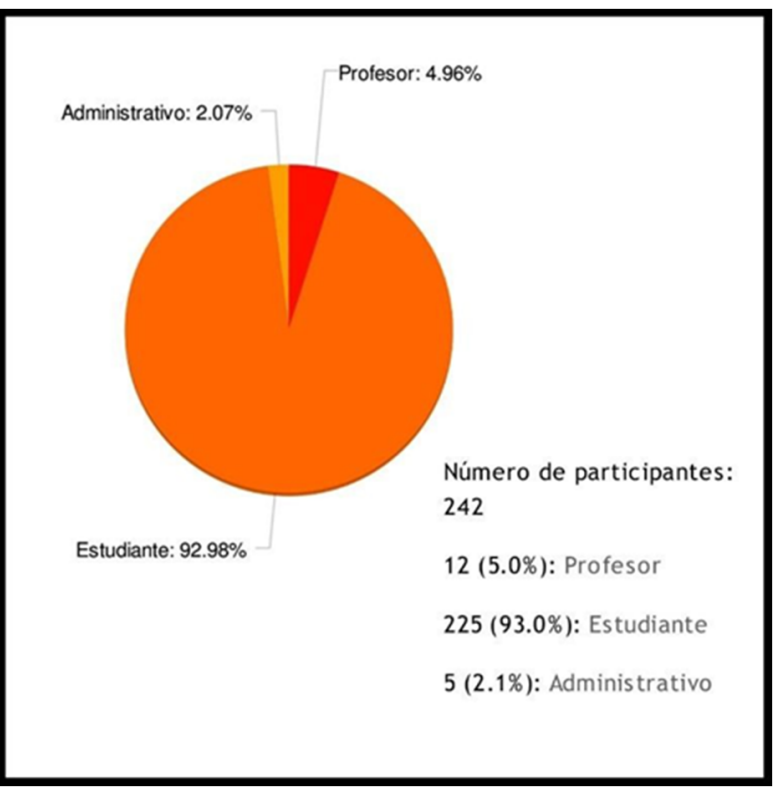

Figura 3 B. Facultad de Ciencias de la Educación.

De ese total (358) el 92.5\% son estudiantes, $5.6 \%$ profesores y $2.0 \%$ son administrativos. (Figura $3 \mathrm{~A}$ ).

En la Facultad de Ciencias de la Educación, la cantidad de estudiantes es de 1,500, 75 profesores y 30 administrativos. La cantidad de encuestados son 242 participantes, en donde 225 son estudiantes (15\% del total de la población), 12 profesores (15\%) y 5 administrativos (15\%).

De ese total (242), en la encuesta el $93.0 \%$ son estudiantes, $5.0 \%$ profesores y $2.1 \%$ administrativos. (Figura 3 B). 


\section{Castañeda (et al): Movilidad urbana en el Campus Central de la Universidad de Panamá: Caso de la Facultad de Arquitectura y Diseño y la Facultad de Ciencias de la Educación}

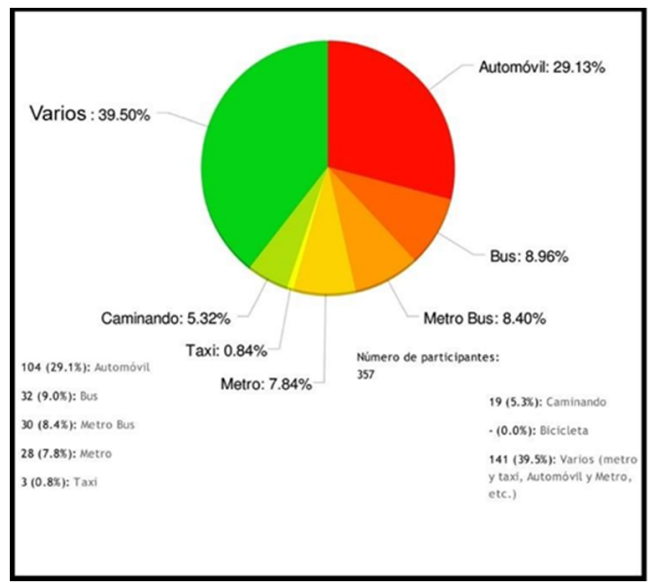

Figura 4 A. Facultad de Arquitectura y Diseño - Tipo de transporte

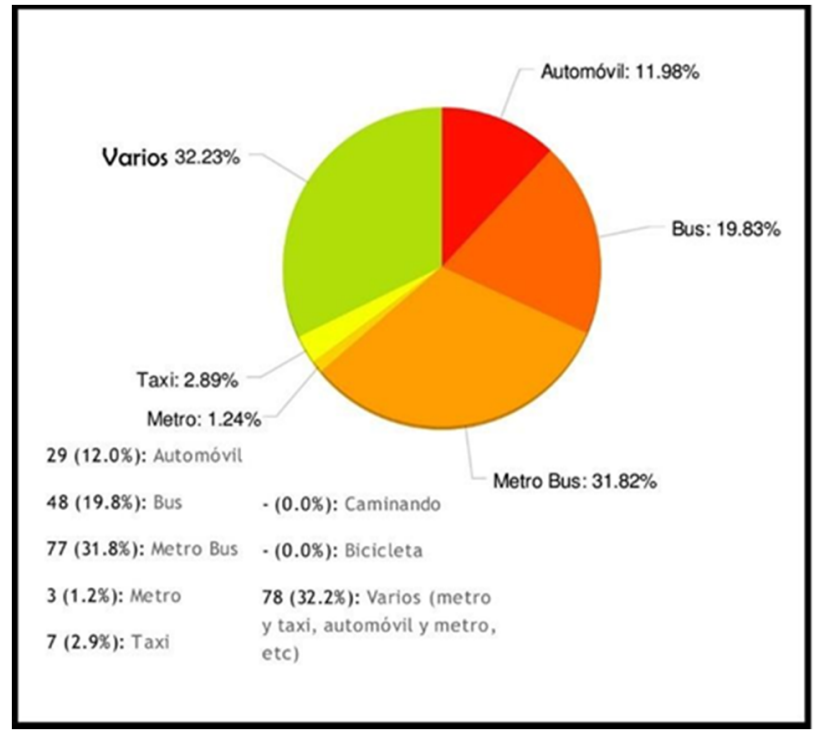

Figura 4 B. Facultad de Ciencias de la Educación.- Tipo de transporte.

Las figuras 4 (A-B), muestra el tipo de transporte empleado por los encuestados de ambas Facultades.

Para la Facultad de Arquitectura y Diseño los resultados en cuanto al tipo de transporte que utilizan los participantes para llegar al campus, fue que un $39.5 \%$ usa varios medios (metro y taxi, bus y carro, carro y metro, etc.), un $29.1 \%$ usa automóvil y un $9.0 \%$ usa el bus. (Figura $4 \mathrm{~A}$ ).

En la Facultad de Ciencias de la Educación los resultados son: un $32.2 \%$ utiliza varios medios (metro y taxi, bus y carro, carro y metro, etc.), el $31.7 \%$ usa el Metro Bus y un $19.8 \%$ usa el bus. (Figura 4 B).

Las figuras (5 A-B), nos muestran el tiempo que se toman en llegar al campus para la Facultad de Arquitectura y Diseño.

Los encuestados tardan hasta 2 horas con un $32.7 \%$, le sigue: Hasta 1 hora con un $31.0 \%$, y un $17.6 \%$ que tarda Hasta 30 minutos. (Figura $5 \mathrm{~A}$ ).

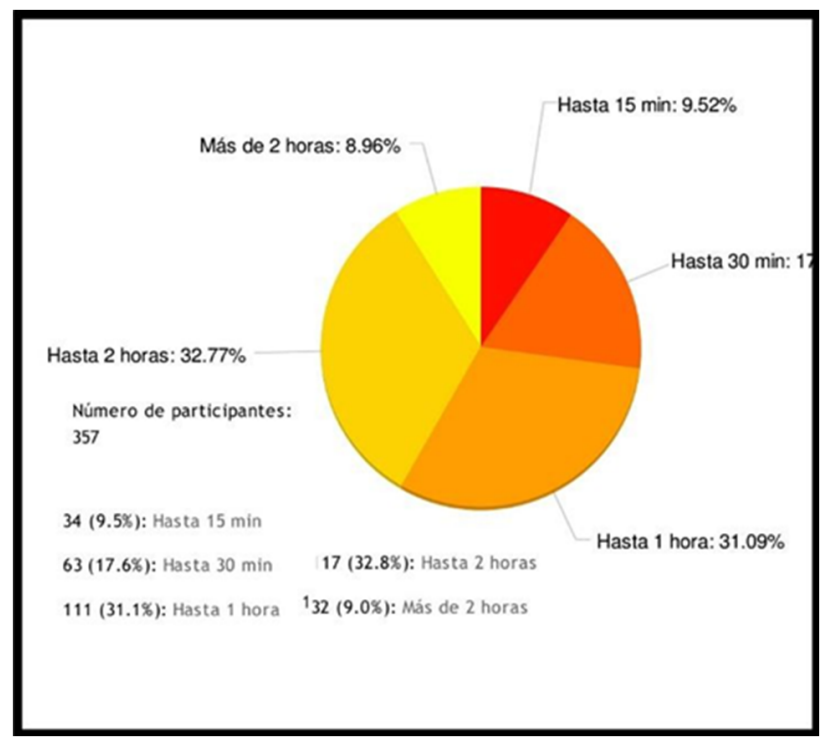

Figura 5 A. Facultad de Arquitectura y Diseño - Tiempo para llegar al campus.

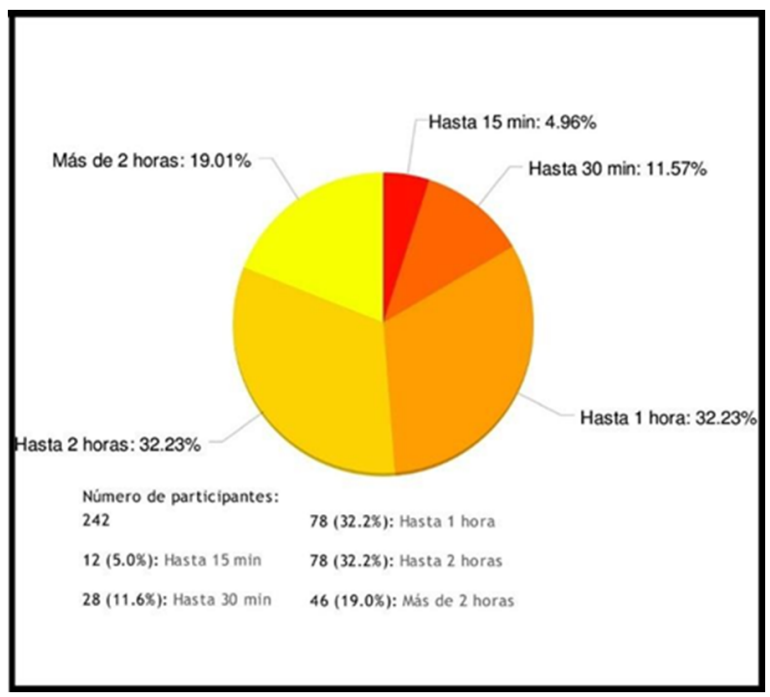

Figura 5 B. Facultad de Ciencias de la Educación - Tiempo para llegar al campus.

Los resultados para la Facultad de Ciencias de la Educación, mostraron que: $32.1 \%$ de los participantes tarda 2 horas, $32.1 \%$ tarda 1 hora y $18.9 \%$ tarda más de 2 horas. (Figura 5 B).

El resultado para saber si los participantes realizan alguna actividad recreativa o de ocio, muestra que para la Facultad de Arquitectura y Diseño, un $63.9 \%$ SÍ realizan actividades recreativas o de ocio fuera de sus casas y un $36.1 \%$ respondió que NO.

Para la Facultad de Ciencias de la Educación el resultado es que un $53.9 \%$ SÍ realizan actividades recreativas fuera de sus casas y un $46.1 \%$ respondió que $\mathrm{NO}$ realizan ningún tipo de actividad. 


\section{Castañeda (et al): Movilidad urbana en el Campus Central de la Universidad de Panamá: Caso de la Facultad de Arquitectura y Diseño y la Facultad de Ciencias de la Educación}

Algunos encuestados comentaban que NO realizaban ninguna actividad extra porque no tenían tiempo o los lugares para que estas actividades se lleven a cabo no eran los correctos y no se sentían inseguros, por lo tanto preferían no hacerlo.

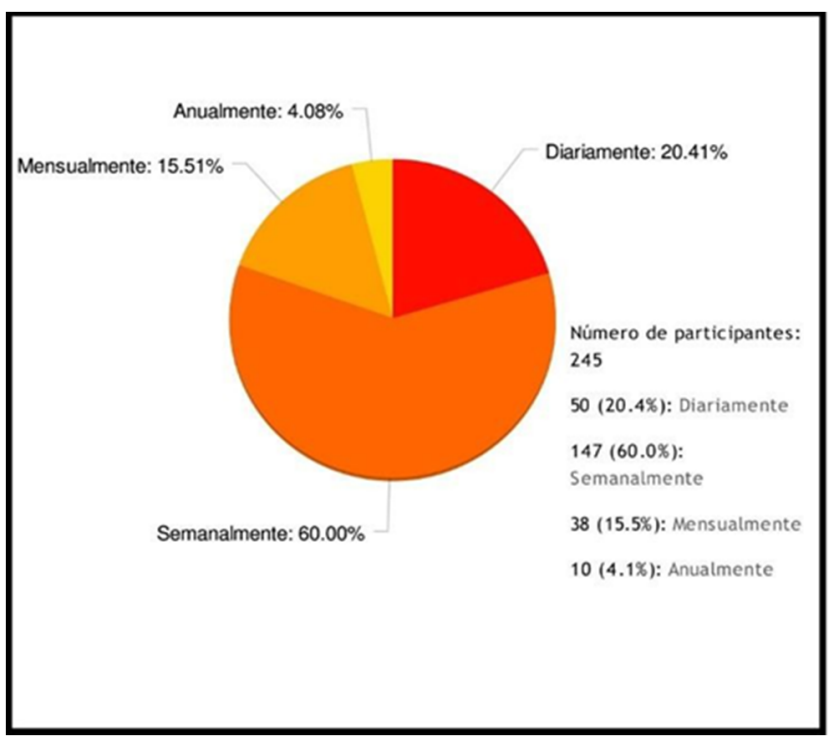

Figura 6 A. Facultad de Arquitectura y Diseño.

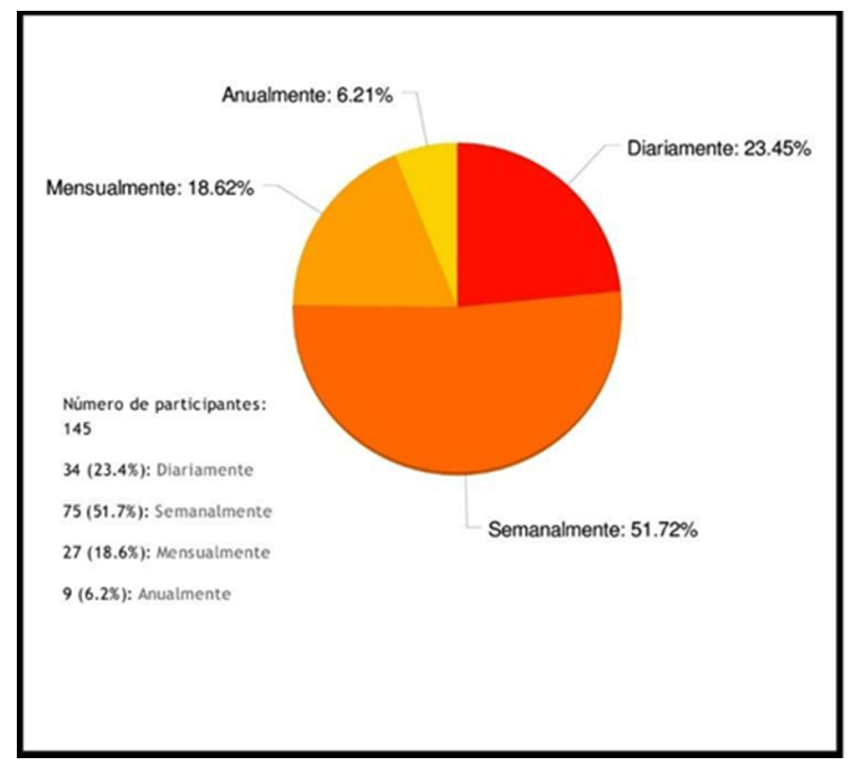

Figura 6 B. Facultad de Ciencias de la Educación.

Las figuras 6 (A- B), nos muestran que el $60 \%$ de los encuestados de la Facultad de Arquitectura y Diseño realizan las actividades recreativas o de ocio por lo menos una vez a la semana y un $20.41 \%$ las realiza diariamente. (Figura 6 A).

En la Facultad de Ciencias de la Educación, un 51.72\% realiza actividades recreativas por lo menos una vez a la semana, mientras que el $23.45 \%$ las realizan diariamente. (Figura 6 B).

Los resultados para los encuestados de la Facultad de Arquitectura y Diseño que realizan actividades recreativas o de ocio, nos muestra que al $46.5 \%$ de los encuestados el sitio de recreación les queda cerca de sus casas, el $35.5 \%$ en un lugar no tan cerca y un $18.0 \%$ el sitio está cerca de la universidad. Para la Facultad de Ciencias de la Educación, los resultados son que un $53.5 \%$ el sitio de recreación queda cerca de sus casas, al $38.7 \%$ no le queda tan cerca y un $7.79 \%$ cerca de la universidad.

En los encuestados en la Facultad de Arquitectura y Diseño los resultados más notables son que un $41.22 \%$ tardan hasta 15 minutos para llegar al sitio donde realizan las actividades recreativas, un $35.92 \%$ que tarda hasta 30 minutos y un $18.78 \%$ hasta 1 hora.

Entre los participantes de la Facultad de Ciencias de la Educación, tenemos que el $39.44 \%$ de los encuestados tardan hasta 15 minutos para llegar al sitio donde realizan sus actividades recreativas, un $23.94 \%$ tarda hasta 30 minutos y un $22.54 \%$ le toma hasta 1 hora.

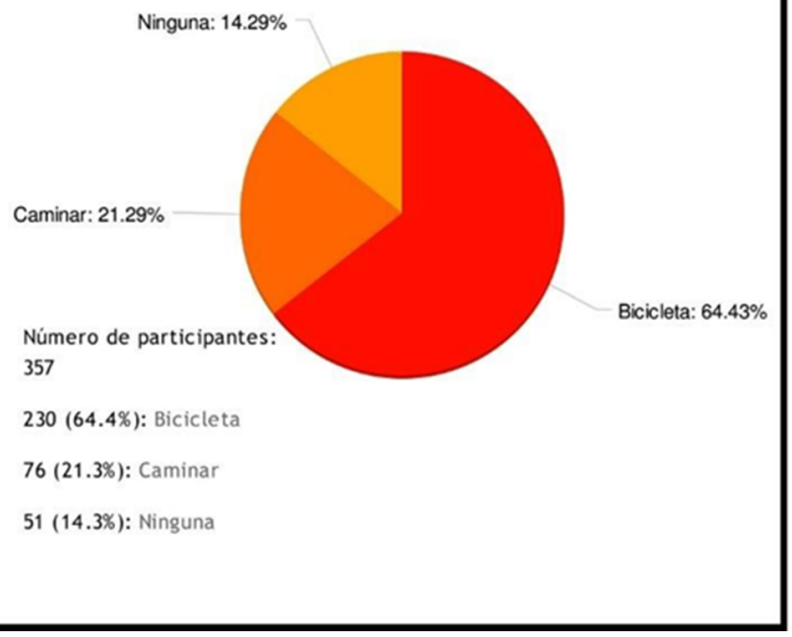

Figura 7 A. Facultad de Arquitectura y Diseño.- Otros medios de transporte. 


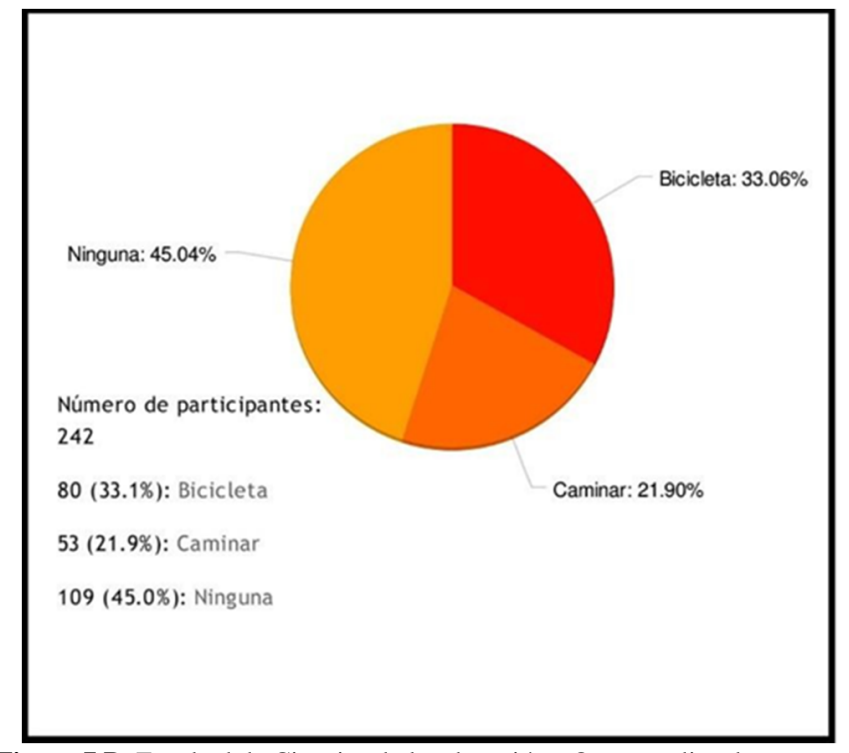

Figura 7 B. Facultad de Ciencias de la educación.- Otros medios de transporte.

La figura 7 A representa la respuesta de si estarían dispuestos a usar algún transporte alternativo en la Facultad de Arquitectura y Diseño. Un 64.4\% de los participantes respondió que utilizarían la bicicleta como medio alternativo de transporte, seguido de un $21.3 \%$ que respondió que caminaría si la ciudad fuera caminable y un $14.3 \%$ no utilizaría ninguna.

Los resultados de la Facultad de Ciencias de la Educación son diferentes a los de la Facultad de Arquitectura y Diseño; de los cuales un $45.04 \%$ no utilizaría ningún medio de transporte alternativo, seguido de un $33.06 \%$ que utilizaría la bicicleta y un $21.90 \%$ prefiere caminar (figura $7 \mathrm{~B}$ ).

\section{Discusión}

En nuestra faena por conseguir los datos de ambas facultades, se presentaron diferentes problemas, uno de ellos fue que la protesta de un grupo estudiantil impidió realizar la encuesta en la Facultad de Ciencias de la Educación en la primera fecha acordada. Debido a esto, se tuvo que definir nuevamente con la dirección de dicha facultad y los profesores una segunda fecha.

También se notó que en la Facultad de Ciencias los estudiantes estaban menos familiarizados con el tipo de preguntas y se les dificultaba responder, pensamos que en un futuro debe hacerse una encuesta más universal, por ejemplo en la pregunta ¿En qué Corregimiento vive? Indicar que si se contestó que vive en Colón o en Panamá Oeste. La siguiente pregunta de los corregimientos no debe ser respondida, también indicar cuales preguntas son obligatorias responder y cuales no o que en algunas la respuesta que se dé, depende de la respuesta que se dio en la pregunta anterior, por ejemplo cuando se pregunta: ¿realizas alguna actividad recreativa o de ocio fuera de tu casa?, la respuesta es SÍ o NO. Si contesta que NO, las siguientes preguntas no se contestarán, y pasarán a la última pregunta directamente y si la respuesta es SÍ, debe responder las preguntas que siguen.

La actitud de las autoridades y profesores de ambas facultades fue muy agradable y abierta. Los profesores cedieron minutos de sus clases para realizar dicha actividad en los salones, incluso los profesores de ambas facultades. Estaban interesados sobre el tema y nos hablaban sobre sus problemas de transporte y la movilización hasta el sitio de trabajo.

En general, los datos que más divergen entre ambas facultades y al mismo tiempo sorprenden, es la respuesta sobre el método alternativo de transporte. Al preguntar a algunos sobre el motivo de su respuesta, decían que no utilizarían ningún medio alternativo, pues al vivir muy lejos del Campus Universitario se les dificultaría caminar o tomar la bicicleta. La gran diferencia en la respuesta de Arquitectura y Ciencias, puede estar en el hecho de que en la Facultad de Arquitectura se habla con más frecuencia sobre los medios de transporte alternativos, los beneficios de usar la bicicleta o caminar, además que los alumnos desarrollan diferentes proyectos donde se diseñan ciclo vías. En general, la concientización a lo largo de los años de aprendizaje puede haber influenciado la respuesta.

\section{Conclusiones}

Los resultados más relevantes de este estudio son:

- La mayoría de la población de la Facultad de Arquitectura y Diseño reside en Bethania (11.48\%), Bella Vista (9.84\%) y Juan Díaz (9.43\%). Mientras que en la Facultad de Ciencias de la Educación, los tres corregimientos con más población en dicha facultad son Bethania (10.13\%), Juan Díaz (10.13\%) y Tocumen $(8.23 \%)$.

- Los encuestados de ambas facultades utilizan en su mayoría varios medios de transporte (como metro y taxi, taxi y bus, automóvil y metro) para transportarse (de sus residencias hasta sus respectivas facultades y viceversa), representando un $39.50 \%$ para la Facultad de Arquitectura y Diseño y un $32.23 \%$ para Ciencias de la Educación.

- En ambas facultades, la mayoría se tarda hasta 2 horas (Arquitectura: $32.77 \%$ y Ciencias de la Educación: 32.23\%) para trasladarse. Cabe destacar que en Ciencias de la Educación un porcentaje significativo (19.01\%) se tarda más de 2 horas.

- En la Facultad de Ciencias de la Educación se utiliza más el Metro Bus (31.82\%) y el bus (19.83\%) que 
en la Facultad de Arquitectura y Diseño donde solo representa un $8.40 \%$ y $8.96 \%$ respectivamente.

- El automóvil es empleado más en la Facultad de Arquitectura y Diseño (29.13\%) que en la Facultad de Ciencias de la Educación (11.98\%).

- En ambas facultades, la mayoría de los estudiantes realizan actividades recreativas (Arquitectura: $63.9 \%$ y Ciencias de la Educación: 53.9\%). Sin embargo, una buena población de ambas facultades respondió que no, pues no tenían el tiempo suficiente. Al verificar la posibilidad del uso de un medio de transporte alternativo (como caminar o la bicicleta) se observó una amplia diferencia entre ambas facultades. Por ejemplo: en la Facultad de Arquitectura y Diseño usarían más la bicicleta (64.43\%) que en Ciencias de la Educación (33.06\%) donde la mayoría (45.04\%) no usarían ningún medio de transporte alternativo.

\section{AGRADECIMIENTO}

Agradecimiento muy sincero a los profesores y profesoras que nos brindaron muy gentilmente tiempo de sus clases para dicha investigación; gracias a Milciades Rodríguez, Saúl Servín, Almyr Alba, Romulo Cerón, José Acosta, Alberto Arosemena y Aracelys.

También, se agradece al equipo del 2016 compuesto por: María Aguilar, Ana Caballero, Katherine Martínez, Débora Sarmiento y Carlos St Omer, quienes en colaboración con SusBCity iniciaron este proyecto de investigación.

También, nuestro agradecimiento a la decana de la Facultad de Ciencias de la Educación, la Dra. Migdalia Bustamante de Avilés, y a los profesores de dicha facultad: Prof. Mercedes, Ostin Osman, Julio Barace, y la Prof. Blanca.

\section{REFERENCIAS}

[1] N. Abbagnano, (1996) Diccionario de Filosofía. México: Fondo de Cultura Económica. México.

[2] A. Pizarro \& J. Kellenberg (2007) El Banco Mundial de Desarrollo La Movilidad Urbana en el Área Metropolitana de Panamá: Elementos para una política Integral. Panamá.

[3] E. A. Vasconcellos, \& A. Mendonça. (2016). CAF Observatorio de Movilidad Urbana: Informe 2015-2016. Caracas.

[4] C. Lange Valdés. (2011). Dimensiones Culturales de la Movilidad Urbana, Revista INVI, 26 (71). 87-106, Chile.

[5] Anónimo (2016), Facultad de Arquitectura y Diseño (figura). Recuperado de: http://www.up.ac.pa

[6] Anónimo, Facultad de ciencias de la Educación (Figura). Recuperado de: http://www.up.ac.pa 